\title{
Identifying 1Method of Meat Containing Excessive Moisture Based on hyperspectral and SVM Multi-Information Fusion
}

\author{
GUO Peiyuan, XU Jingjing, XU Pan, DONG Xiaodong, LIU Yanfang \\ XING suxia, SUN mei \\ College of Computer and Information Engineering, Beijing Technology and Business University, Beijing, China,Email:ggppyy@126.com \\ Project 61473009 supported by NSFC
}

\begin{abstract}
In this paper, a quick and accurate detection method which can identify whether the meat contain excessive moisture is mentioned. By using near-infrared spectroscopy measurement and SVM Multi-Information Fusion, the meat moisture content model has been established. In order to improve the accuracy of NIR measurement predicted model and to reduce the measurement sensitivity, utilizing image information and the $\mathrm{PH}$ value data as the parameters of the meat moisture content model. The study concluded that the theory and method can be further extended to the detection of other related meat agricultural products.
\end{abstract}

\section{Introduction}

In recent years, food safety issues have attracted more and more attention, at the same time, the event "water-injected meat" which shocked consumers. Waterinjected meat is the low-quality meat that illegal in pork and beef. The national standard meat moisture limit as follows: if the pork, beef, chicken, water content is more than $77 \%$, can be judged as the meat injection. In the present stage, method of detecting water-injected meat has two main kinds: sensory detection (i.e. through view color) and physicochemical detection. Sensory evaluation methods can be used to quickly determine the meat, but the accuracy is poor; Physical and chemical detection method can obtain accurate results, but the chemical experiment needs for a long time.

With the development of information technology, NIR spectrum detection, computer vision, multiinformation data fusion detection technology used in the food safety which can provide a rapid, effective and practical non-destructive testing methods for meat quality. so this paper use the moisture content of the meat as a main parameters, use the color value and the $\mathrm{pH}$ value of the meat as the auxiliary parameter parameters to identify the water-injected meat, to extract the characteristics of these three data and carry on the multiple information fusion.

\subsection{The Model of NIR for Water Content in Meat}

NIR technology is the physical measurement techniques that it utilizes chemical properties in the near infrared region to rapidly determine content and property of one or more chemical components in the sample [1]. When using near infrared spectroscopy for determining the content of ingredients in the meat, usually chopped

\footnotetext{
a Corresponding author: ggppyy@126.com
}

the meat and stir till to uniform mixture, so that it can accurately determine the content. This study uses FoodScan NIR Spectrometers of the FOSS Company for measuring meat spectrogram.

FoodScan NIR Spectrometers (shown in Fig.1) is a fast, accurate and easy to use instrument for analysing all stages of meat production - from checking incoming raw material to final product control. It is pre-calibrated to analyse all key parameters including fat analysis, moisture analysis, protein analysis, salt analysis and collagen analysis delivering results in just 50 seconds.

In order to eliminate the interference of environmental light to lighting equipment, the illumination system adopts the closed box with $400 \mathrm{~mm} \mathrm{x}$ $400 \mathrm{~mm}$ square at the bottom. Box is made of white aluminum-plastic plate, the inner wall are white, the top two adjustable halogen light source. At the top of the box for the camera hole, placed the digital camera. Right below of the camera hole is the elevating pallet for carrying the measured meat. The illumination value of the whole measured meat can be adjusted by adjusting the height of the tray. The structure diagram of light box is shown in Fig.2.
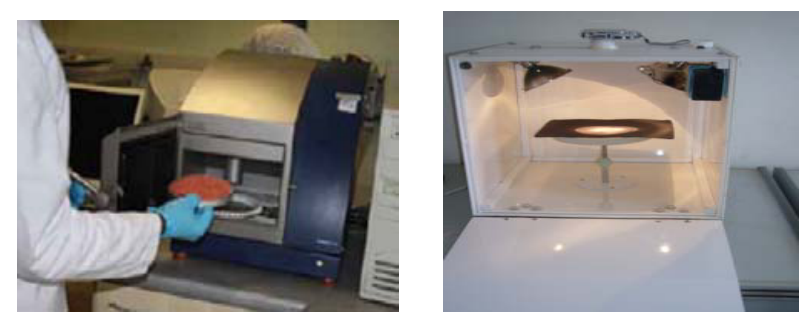

Figure 1. FoodScan NIR Figure 2. Image acquisition system Spectrometers 


\subsection{Spectral Data Acquisition and Preprocessing}

In the experimental environment at room temperature $25^{\circ} \mathrm{C}$, the sample set include 30 group normal meat samples and 30 group water-injected meat samples. Spectra collected and then completed each sample with a control experiment, which uses physicochemical method for measuring the moisture content of meat. In the MATLAB7.11.0 (R2010b), generated data file, viewed the sample group 1-40 as the training set and the sample group 41-60 as the test set for the predictive mode. After analysis and detection, moisture content in water-injected samples is more than $77 \%$, in line with national standards, which can be used as water-injected meat samples for experimental research. The statistical result of the experimental data was shown in Table 1.

Table1: The moisture of training and testing set for the NIR mode

After repeated experiments, studies have adopted the multiple scatter correction (MSC) and centralized method to do pretreatment for the spectral data.

Multiplicative scatter correction (MSC), not only can effectively remove the uneven distribution of the sample particles and the particle size generated by scattering effects in the near-infrared spectrum, but also can modify a path length error in the measurement of the transmission spectrum.

Specific calculation method as follows:

(1) Calculate the average spectrum of the spectra of the samples collected:

$$
\bar{x}=\frac{\sum_{i=1}^{n} x_{i}}{n}
$$

(2) Calculate regressively for the above average spectrum:

$$
x_{i}=a_{i} \bar{x}+b_{i}
$$

(3) Make multiplicative scatter correction (MSC) calculation for each spectrum:

$$
x_{i, M S N}=\frac{x_{i}-b_{i}}{m_{i}}
$$

The mean spectrum centralized method is that the average spectrum of near infrared spectroscopy subtract the average spectrum of the calibration set and make the calibrating spectral matrix X column average to be zero; so the modeling is more suitable for the meat water content.

Specific calculation method as follows:
(1) Calculate the average spectrum of the calibration set of samples:

$$
\bar{x}_{j}=\frac{\sum_{i=1}^{n} x_{i j}}{n}
$$

In this structure: $\mathrm{n}$ is the number of calibration samples, $j=1,2, \ldots, m$

(2) Calculate the mean center spectrum $x_{i}(1 \times m)$ of the unknown sample spectra $x_{i, \text { mean }}$ :

$$
x_{i, \text { mean }}=x_{i}-\bar{x}
$$

\subsection{BiPLS Method for Prediction Model}

Backward interval partial least squares (BiPLS) method for prediction model In MATLAB7.11.0 (R2010b), the spectral information of training set sample is shown in Fig.3.

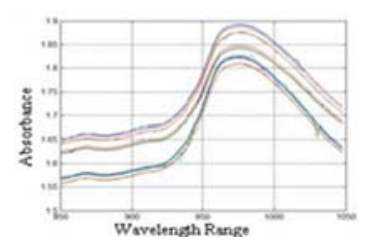

Figure 3.Spectral of training set samples
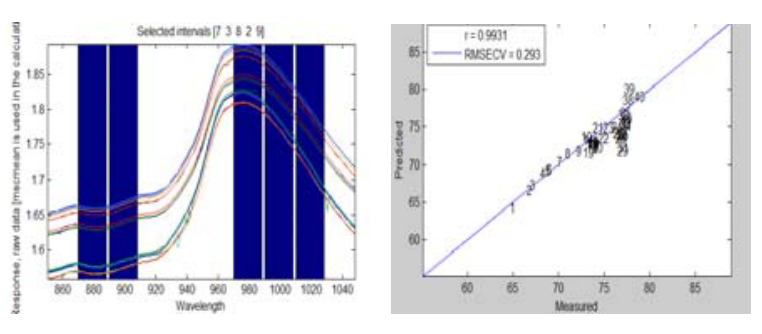

Figure 4.Select the best subintervals of the model

According to the minimum root mean squared error correction (RMSEC) principle of BiPLS algorithm (shown in Fig.4). choose 5 subintervals, including number 2, 3, 7, 8, 9, to establish PLS model. The root mean square error of cross validation (RMSECV) of the PLS models, RC, RP, root mean square error of prediction (RMSEP) were 0.9821, 0.442, 0.9985, 0.318 . The number of variables which the model optimization needed is reduced by half and the prediction performance is good. In order to improve the accuracy of model, make a comprehensive evaluation to the meat, in this paper, by adding auxiliary variables $\mathrm{pH}$ value and meat color information for multi-data fusion detection, optimize the prediction model which has been established.

\section{The Extraction of Meat Color Information}




\subsection{The Image Pretreatment and Color value Extraction}

When collected images in the light box and would extract the color information of the image processing. Identifying the color of the meat with the computer vision technology is the use of the RGB color and HSI color model. The color characteristic value adopted in this study is the mean and standard deviation of the R, G, B and $\mathrm{H}, \mathrm{S}, \mathrm{I}$ component. Wherein the mean value of the color indicates the basic color of the meat, the standard deviation of the color indicates the uniformity of the meat color. In the MATLAB image processing toolbox, using the following procedures can obtain the R, G, B component of image:

$\mathrm{R}=$ photo $(:,:, 1) ; \mathrm{G}=$ photo $(:,:, 2) ; \mathrm{B}=$ photo $(:,:, 2)$;

According to the RGB to HSI conversion equation, In the MATLAB can extract $\mathrm{H}, \mathrm{S}$, I component:

$$
\begin{gathered}
H=\arccos \left\{\frac{[(R-G)+(R-B)]}{\left[2(R-G)^{2}+(R-B)(G-B)\right]^{1 / 2}}\right\} \\
S=1-\frac{3}{(R+G+B)}[\min (R, G, B)] \\
I=\frac{1}{3}(R+G+B)
\end{gathered}
$$

By experiment and analysis, there are some differences in the color between the normal meat samples and the water-injected meat samples (shown in Fig.5 and Fig.6) ; The normal meat sample is cherry red and with rich color, the color of water-injected meat samples is light pink because it injected a large number of moisture.

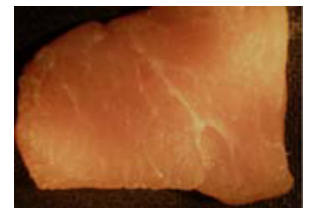

Figure 5. for normal meat Figure 6. excessive moisture meat

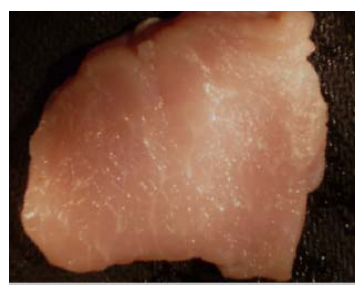

Figure 7.pH value of the meat

Therefore, the color characteristic values $(\mathrm{R}, \mathrm{G}, \mathrm{B}$ and $\mathrm{H}, \mathrm{S}, \mathrm{I}$ ) of water-injected meat samples, can be used as one of the basis to judge whether it is water-injected meat.
The $\mathrm{pH}$ value is one of the important quality the meat, which influences the color, water-lockup, and the quality of the meat [3]. This paper adopts ray magnetic PHS-3D $\mathrm{pH}$ meter to measure the $\mathrm{pH}$ value of the meat, As shown in the figure.7.

before collecting the data need to break the meat to pieces. The electrode was inserted into the sample, record the value after mixing evenly, and repeat this operation until the record of $\mathrm{pH}$ value is stable. The statistical results of experimental data collected in this study are shown in Table 1.

Table 1: Measurement results of $\mathrm{pH}$

\begin{tabular}{|c|c|c|c|c|}
\hline PH value & $\begin{array}{c}\text { Sample } \\
\mathrm{s}\end{array}$ & Maximum & $\begin{array}{c}\text { Minimu } \\
\mathrm{m}\end{array}$ & Variance \\
\hline Training set & 40 & 5.66 & 5.32 & 0.01 \\
\hline Testing set & 20 & 5.70 & 5.31 & 0.01 \\
\hline
\end{tabular}

There is obvious difference in $\mathrm{pH}$ value between the normal meat and the water-injected meat, is the meat $\mathrm{PH}$ measurements shown in Fig.8. The $\mathrm{pH}$ of normal meat (pork through muscle) is generally greater than 5.4. Whereas the water-injected meat due to injection of a large number of moisture, make further loss of watersoluble substances (such as amino acids) in the meat and then lower the $\mathrm{pH}$ value. As a result, according to the trend of changes in the $\mathrm{pH}$ value can identify whether the meat is water-injected meat.

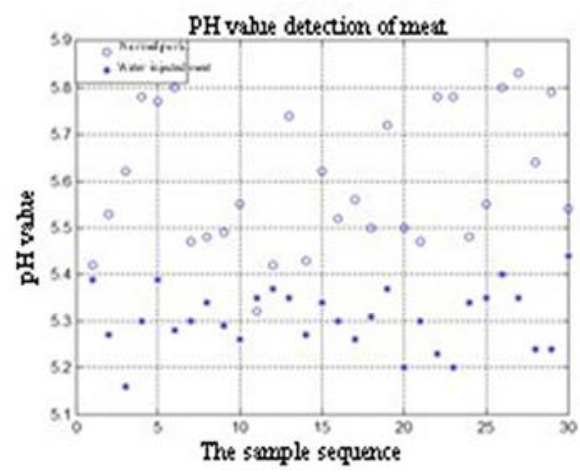

Figure 8. Measurement results of $\mathrm{pH}$

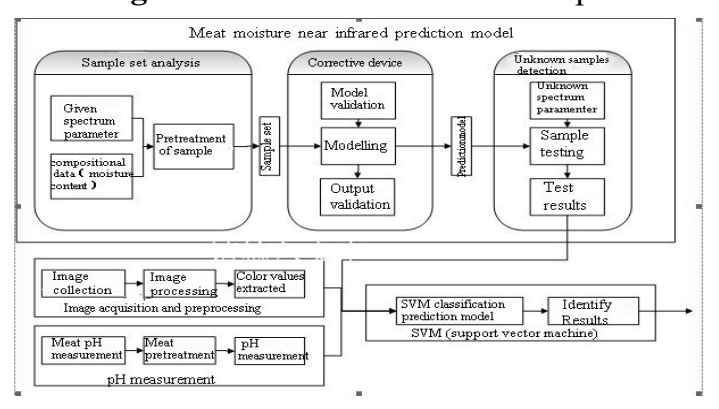

Figure 9. Support Vector Machine (SVM) prediction model

\subsection{Measurement of PH Value of Meat}




\section{The Establishment of Multi- information Fusion}

\subsection{Support Vector Machine (SVM) Prediction Model}

Although the use of near infrared spectroscopy can be used to predict the moisture content of meat, this method is not the original method and need constant maintenance. In order to guarantee the accuracy, this paper adopts the method of the multi-information fusion, using the moisture content of meat detected by NIR, the mean color value $(\mathrm{H}, \mathrm{I}, \mathrm{S}, \mathrm{R}, \mathrm{G}, \mathrm{B})$, the standard deviation obtained by the image acquisition system and the $\mathrm{pH}$ value measured as input features, adopting the SVM algorithm for multi-information fusion, to establish a water-injected identification model. The overall structure of this study is shown in Fig.9.

As shown in the figure.6, the moisture infrared predictive model added the structure of correction for improving the detection accuracy; and then the moisture value and the value of color and the $\mathrm{pH}$ value as input values of SVM classification prediction model.

$$
f(x ; w, b)=\operatorname{sign}\left(w^{T} x+b\right)
$$

$$
G(x, z)=\varphi^{T}(x) \varphi(z)=\sum_{j=0}^{M} \varphi_{j}(x) \varphi_{j}(z)
$$

$\left|\mathrm{y}_{i}\left(w^{T} x_{i}+b\right)\right| \geq 1, i=1,2, \ldots, k$

In summary, using the mean and standard deviation of meat color value, $\mathrm{pH}$ value and the meat water content, a total of 14 characteristic component as input features for the support vector machine (SVM) classification prediction model, and using the 60 groups samples collected by experiment as sample set, which include 40 groups samples as the training set and 20 groups samples as the testing set; Namely, a total of 60 samples, each sample contains 14 characteristic component. Due to the choice of the integral operator kernel functions directly affect the performance of the classifier, so use a variety of the integral operator kernel function to establish the model and compare the results.

\subsection{Support Vector Machine (SVM) Prediction Results}

In the MATLAB7.11.0 ( R2010b ), generated data.mat file by used the collected data, then viewed The sample group 1-40 as the training set and the sample group 41-60 as the test set for the predictive mode. The lib-svm toolbox [5] is adopted to establish the SVM classifier prediction model, and select the parameter $\mathrm{c}=1$, $\mathrm{g}=4$; the result is shown in Fig.10. According to statistics, the results Accuracy $=95 \%$, can meet the research requirements, and realize the fast discrimination whether the meat is the water-injected meat.

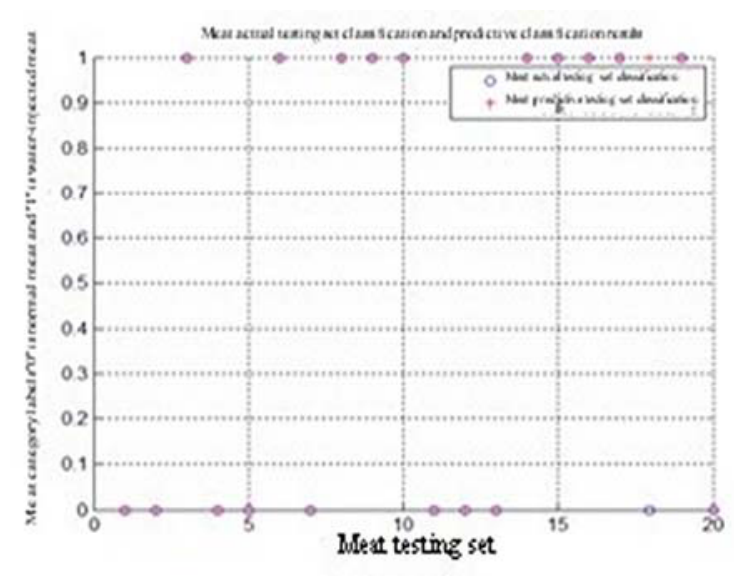

Figure 10. Support Vector Machine (SVM) prediction results

\section{Conclusion}

In this paper, used the NIR technique to measure the moisture content of the meat; In order to improve the NIR measurement precision and accuracy and overcome the meat moisture measurement is affected, the color value and the $\mathrm{pH}$ value of the meat as the auxiliary parameter was adopted, and then established and optimized the multi-information fusion model. As experiments shown, the method used in this paper compared to the other two common methods this study (shown in Table 2), can be in a relatively short period of time to make quick and accurate judgment whether the meat is water-injected meat.

\begin{tabular}{|c|c|c|c|}
\hline $\begin{array}{l}\text { Detectio } \\
n \\
\text { methods }\end{array}$ & $\begin{array}{l}\text { Sensory } \\
\text { evaluatio } \\
n \text { method }\end{array}$ & $\begin{array}{l}\text { Physical } \\
\text { and } \\
\text { chemical } \\
\text { detection } \\
\text { method }\end{array}$ & $\begin{array}{l}\text { Method in } \\
\text { this paper }\end{array}$ \\
\hline $\begin{array}{c}\text { Advanta } \\
\text { ge }\end{array}$ & $\begin{array}{c}\text { Convenie } \\
\text { nt and } \\
\text { simple }\end{array}$ & Accurate & $\begin{array}{l}\text { Convenient } \\
\text {, simple } \\
\text { and } \\
\text { accurate }\end{array}$ \\
\hline $\begin{array}{c}\text { Disadvan } \\
\text { tage }\end{array}$ & $\begin{array}{l}\text { Complete } \\
\text { ly relies } \\
\text { on the } \\
\text { experienc } \\
\text { e and } \\
\text { with } \\
\text { unilateral } \\
\text { results }\end{array}$ & $\begin{array}{c}\text { Operation } \\
\text { is } \\
\text { complex, } \\
\text { time } \\
\text { consumin } \\
\text { g, } \\
\text { destructiv } \\
\text { e, }\end{array}$ & $\begin{array}{c}\text { Equipment } \\
\text { support }\end{array}$ \\
\hline Time & $\begin{array}{l}\text { About 2- } \\
3 \text { minutes }\end{array}$ & $\begin{array}{c}\text { Take a } \\
\text { long time }\end{array}$ & $\begin{array}{c}\text { About } 4-5 \\
\text { minutes }\end{array}$ \\
\hline
\end{tabular}

Table 2: The comparison results of three detection methods

consequently, the method can realize rapid and accurate detection of water-injected meat. The health and quarantine departments and related meat manufacturers can use this fast, accurate, practical detection technology to detect the water-injected meat, eliminate unqualified meat in the first time and ensure the food safety. 
The main author, Guo Peiyuan (1958 -), professor and Ph.D. is mainly engaged in researching of intelligent detection technology of agricultural products and multiple data fusion, embedded technology.

\section{Acknowledgement}

This work is supported by National Natural Science Foundation (NNSF) of China under Grant 61473009.

The main author, Guo Peiyuan (1958 -), professor and $\mathrm{Ph}$.D. is mainly engaged in researching of intelligent detection technology of agricultural products and multiple data fusion, embedded technology.

\section{References}

1. Sun,Mei, Fu, Yan , Xu Ranran etc. Non-destructive testing of fruit quality based on hyperspectral imaging technology. Journal of Food Technology Science,31(2):67-72,2013.

2. Guo, Peiyuan. Bi Song, Chen, Tianhua, Xu, Guannan, Liu, Xing The fresh classification of pork detection based on multi-data fusion, 29th Chinese Control Conference 2010(7); 213-316

3. Zhao Jiewen, Zhang Haidong, Liu Muhua. Nondestructive Determination of Sugar Content in Apples Using Near Infrared Diffuse reflectance [J]. Transaction of the CSAE, 2010, 21 (3):163-165.

4. Andrews B S, Huntchison S, J. A. Journal of Muscle Foods, 2007, 18:

5. Shi Feng, Wang Xiaoshuan, YuLei et al. MATLAB Neural Network Analysis of 30 Cases[M]. Beijing: Beijing University of Aeronautics and Astronautics Press, 2011.

6. Guo Peiyuan, Chen Yan, Xuan Hong, et al. The Device on Intellectual Detection Techniques to Freshness of Pork. China, 101144780A [P]. 2008,03,19.

7. Zou Xiaobo, Zhao Jiewen, Xia Rong. Near infrared Determination of Sugar Content in Apples Based on Multi-resolution Decmpositiion and Interval Partial Least Square (IPLS ) Method[J]. Transactions of the Chinese Society for Agricultural Machinery, 2010,37(6)79-82.

8. Li Xi. Research and Soft Sensing Applications of Partial Least-squares Regression [D]. Dalian: Dalian University of Technology. 2011.

9. Chen Bing, Li Shan, Wu Baohai et al. Multistage Manufacturing Quality Analysis and Forecasting on Dimension Association and Partial Least Square Regression. CIMS, 2010, 15(2):389-398.

10. Peiyuan Guo , Yan, Fu , Fang, Yuan, Man, Bao Research on intellectual detection and classification of pork freshness based on SOM neural network, Mechatronics and Automation 2011.8:432-435

11. Fu yan, Guo Peiyuan, Sun mei Study on Meat Quality Detection Techniques, Bioinformatics and Biomedical Engineering 2012.5:35-37

12. Panigrahi S, Balasubramanian S, GuH. Neuralnetwork-integrated electronic nose for identification of spoiled beef[ J]. LWT-Food Science andTechnology, 2012, 39: 135-145.

13. [13] Fu,Yan,Guo,Peiyuan, Sun,Mei,etc. Meat Quality Detection Technology Review. Journal of Beijing Technology and Business University (Natural Science),30(1):69-71,2012 